\title{
OBSERVATION OF THE SOLAR ECLIPSE OF 30 JUNE 1954 AT 9.4 CM. WAVE-LENGTH
}

\author{
C. H. MAYER, R. M. SLOANAKER AND J. P. HAGEN \\ Naval Research Laboratory, Washington, D.C., U.S.A.
}

The observation of the total solar eclipse of 30 June 1954 at $9.4 \mathrm{~cm}$. wavelength was one of the experiments carried out by the Naval Research Laboratory eclipse expedition to Sweden. The apparatus was set up in the city of Oskarshamn at a point about $5 \mathrm{~km}$. north of the centre-line of totality. At this location the eclipse was total, with magnitude $\mathrm{I} \cdot 035$, at ${ }_{1} 2^{\mathrm{h}} 4^{8^{\mathrm{m}}}$ U.T.

A Dicke-type radiometer using a paraboloidal reflector $\mathrm{I} \cdot 83$ metres in diameter was mounted on a polar axis and motor driven to track the sun during the eclipse. The angular width of the antenna pencil-beam was about 3.5 . The superheterodyne receiver had an average noise factor of 7 and the band-width of the intermediate-frequency amplifier was $5.5 \mathrm{Mc}$./s. The power from the antenna was interrupted thirty times a second with an absorber and the resulting modulation was detected in a coherent detector circuit. The output time constant was about one second. For the eclipse measurement, the modulation-frequency output was amplified and detected in two separate channels. The output of each channel was displayed by two recording meters. Four simultaneous, semi-independent records of the receiver output were obtained.

During three weeks preceding the eclipse the characteristics of the apparatus and the operating conditions at the site were evaluated. On the day of the eclipse, a set measurement procedure was followed from one hour before first optical contact until one hour past fourth optical contact. The radiation from the sun was monitored continuously except for intervals of about two minutes duration when reference points were inserted into the output records. The reference points were of four types: the output when a thermal noise source at about $400^{\circ} \mathrm{K}$. was substituted for the antenna, the output when a thermal noise source at about $295^{\circ} \mathrm{K}$. was substituted for the antenna, and the outputs when the antenna was pointed to declinations $8^{\circ}$ above and below that of the sun. The four 
reference-point measurements were repeated at 15 or 20 minute intervals and were spaced from each other by 2-minute measurements of the radiation from the sun.

The information obtained during the eclipse is illustrated in Fig. I, which shows the data from one of the recorders. The variation in the gain of the receiver was calculated from the measurements of the two thermal noise sources and is plotted in the upper box. The uncertainty in the gain measurement is of this same order. The receiver output information is plotted in the lower box. The heavy line represents the uncorrected output meter readings when the antenna was pointed at the sun. The measure-

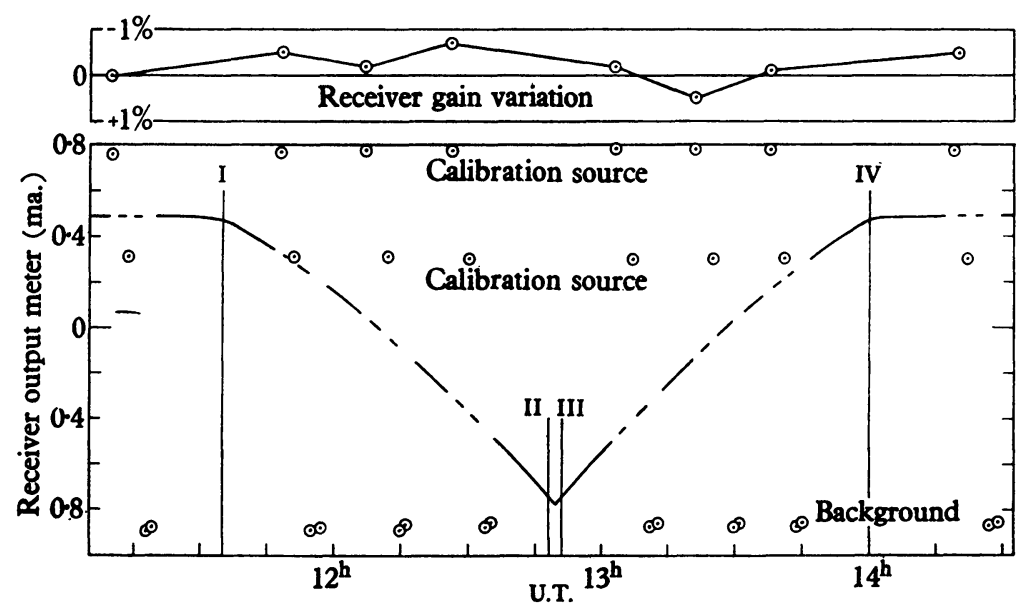

Fig. I. Total eclipse of 30 June 1954 at $9 \cdot 4 \mathrm{~cm}$. wave-length (uncorrected data-recorder no. I).

ments of the thermal noise sources and of the background level above and below the sun are enclosed in circles. The data through which the eclipse curve was plotted are smooth and symmetrical to within I \% of the uneclipsed solar flux level.

The data from the four simultaneous records of the eclipse agreed to less than I $\%$. The radiation from the sun as measured by all four recorders is plotted in Fig. 2. The two halves of the eclipse curve have been normalized separately and superimposed. The dots represent points from the first half of the eclipse curve and the crosses the second half. It was not possible to represent all the points with strict accuracy, but a good indication is given of the agreement between the four records and the smoothness of the change in solar flux during the eclipse. The solar radio radiation decreased smoothly during the eclipse to a minimum value of about $7 \%$ of the flux measured before and after the eclipse. 


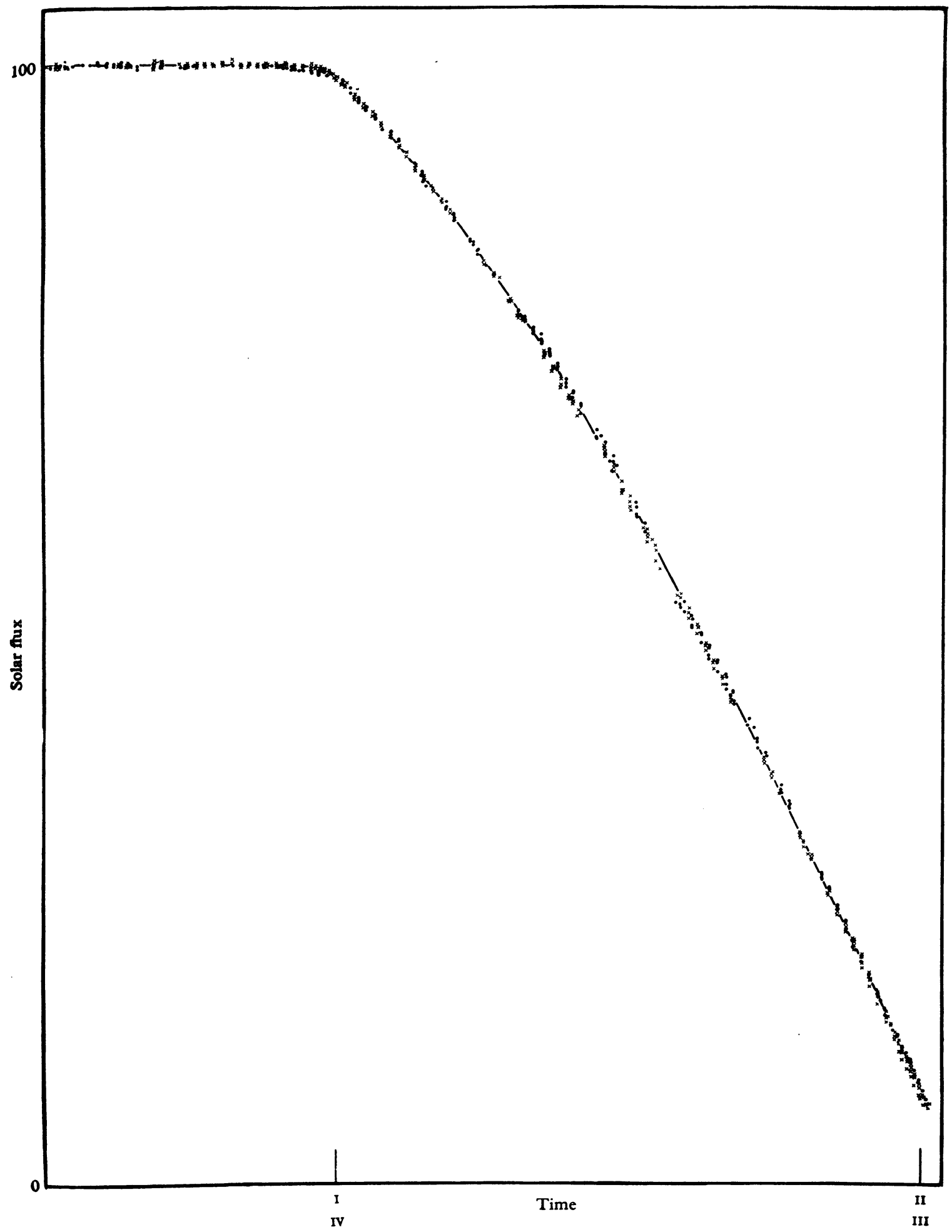

Fig. 2. The $9 \cdot 4 \mathrm{~cm}$. eclipse curve, 30 June 1954, Oskarshamn, Sweden. 
The smoothness and symmetry of the eclipse curve indicate that no appreciable part of the radiation from the sun originated in a localized region emitting intense radio radiation. This indication of a very low level of solar activity is supported by the absence of active regions on the visible hemisphere of the sun, as reported by the solar observatories, and by the measurements of solar radio radiation. The low level of solar activity increases the value of a measurement of this eclipse as a control over models of the undisturbed solar chromosphere.

A radial brightness distribution for the sun at $9.4 \mathrm{~cm}$. wave-length has been calculated from the eclipse measurement under the assumption of spherical symmetry. The curve showing this distribution is reproduced in Fig. 2 of the following paper (p. 276). It has the form with limbbrightening predicted by quiet-sun theory under the same assumption of symmetry. The eclipse curve corresponding to the brightness distribution, shown on p. 276 , agrees with the measured eclipse curve to within one percent. A redistribution of a small percentage of the total radiation to the equatorial limb regions gave a slightly improved comparison to the measured eclipse curve. A definite brightening near the limb of the sun is shown in both cases. 